\title{
Medidas generales en Dermatología
}

\section{General Measures in Dermatology}

Luis Angel Pérez-Buenfil, Luz Orozco-Covarrubias, Marimar Sáez-de Ocariz

\section{Generalidades}

La piel funciona como barrera entre el cuerpo y el medio ambiente al prevenir la pérdida de líquidos y electrólitos, regular la temperatura corporal y proteger contra infecciones y diversos estímulos mecánicos, térmicos y ambientales. Además, es esencial para el sentido del tacto y la síntesis de vitamina D. ${ }^{1-4}$

En el niño, la piel es frágil, delgada y más propensa a dermatitis e infecciones..$^{2,5,6}$ En el adolescente, la piel experimenta cambios secundarios al alza en los andrógenos circulantes, que se traducen en mayor producción de sebo y predisposición al acné.

Las medidas generales son un conjunto de cuidados universales de la piel que favorecen su aspecto saludable y previenen la aparición de diversas dermatosis. Entre ellas se incluyen: recomendaciones acerca del baño, la hidratación cutánea, los textiles y la fotoprotección. Cuadro 1

Baño

El baño tiene la finalidad de eliminar impurezas, bacterias y contaminantes. Debe realizarse con agua tibia, de manera gentil y cuidadosa, sin tallar la piel. ${ }^{8}$ Los jabones alcalinizan el pH cutáneo, alteran la función de barrera e irritan la piel; ${ }^{9}$ por ello, se recomienda utilizar limpiadores con fórmulas no irritantes e hipoalergénicas, como los jabones con pH neutro o ácido, o sustitutos de jabón. ${ }^{10-13}$

El secado debe practicarse de forma suave y sin frotar, pues la fricción generada ocasiona que se adelgace el estrato córneo y disminuya la capacidad de retención de agua en la piel. ${ }^{14}$

\section{Hidratación cutánea}

Hidratante es un término genérico que engloba a humectantes, emolientes y oclusivos. Un humectante es una sustancia que atrae agua desde la dermis hacia la epidermis y el estrato córneo, en tanto que el
Servicio de Dermatología.

Instituto Nacional de Pediatría, Ciudad de México.

Recibido: 20 de octubre 2020

Aceptado: 30 de noviembre 2020

Correspondencia Marimar Sáez-de-Ocariz

mmsaezdeocariz@gmail.com

Este artículo debe ciatrse como: Pérez-Buenfil LA, Orozco-Covarrubias L, Sáez-de Oscariz M. Medidas generales en Dermatología. Acta Pediatr Méx 2021; 42 (1): 44-7. 
emoliente "encierra" el agua en la epidermis y el estrato córneo para que la piel tenga un aspecto suave y liso. Los emolientes oclusivos contienen ingredientes que crean una barrera física sobre el estrato córneo previniendo las pérdidas transepidérmicas de agua. Con frecuencia se combinan los tres en una misma formulación. ${ }^{15,16}$

La hidratación contribuye a mejorar la función de barrera, promover la reparación y reducir la susceptibilidad a lesiones de la piel..$^{15,16} \mathrm{El}$ uso diario de emolientes reduce de manera significativa el riesgo de padecer dermatitis atópica hasta en $50 \%$ en pacientes con factores de riesgo. ${ }^{17}$

La recomendación habitual es la aplicación de crema blanca, sin perfumes, una o dos veces al día, en la piel del tronco y las extremidades.

Cuadro 1. Medidas generales

Baño diario con jabones suaves, agua tibia y sin tallar la piel.

Secado gentil sin frotar la piel.

Uso de crema hidratante 1 o 2 veces al día (más en caso necesario).

No aplicar perfumes directamente sobre la piel.

Usar preferentemente ropa de fibras naturales y delgadas, y ropa suelta.

Uso diario de protector solar con FPS de al menos 30+.

En adolescentes:

- Lavado de cara dos veces al día en forma gentil

- Uso de cosméticos hipoalergénicos, libres de aceite, no comedogénicos y fáciles de retirar

- Para el afeitado: lavado de cara, aplicación de gel para afeitar $y$, luego, crema hidratante

\section{Textiles}

Los textiles están en contacto con la piel durante largos periodos. Por ello pueden jugar un papel en la etiología de algunas dermatitis. ${ }^{18}$ Las fibras más utilizadas para la ropa son las sintéticas, como poliamida o poliéster, y las naturales como algodón, seda y lana, entre otras. Se prefiere el uso de telas naturales, hidrofílicas, de fibras del- gadas, como el algodón, la seda o la lana merino, con bajo potencial irritante y con propiedades termorreguladoras (reducen la absorción de calor) y de transporte de humedad (retienen entre 25 y $35 \%$ de su peso en agua).

Es importante reducir la sudoración y favorecer el uso de ropa suelta porque las áreas con mayor sudoración y fricción y en contacto más estrecho con la ropa son más propensas a la dermatitis por contacto. La humedad generada en estas zonas facilita la liberación de tintes y resinas con potencial alergénico, desde la ropa o sus etiquetas. ${ }^{19-22}$

\section{Fotoprotección}

La fotoprotección es el conjunto de medidas que pueden aplicarse para proteger la piel de la agresión producida por la exposición a los rayos solares. ${ }^{23}$ Se aconseja en todas las edades, pero debe tenerse especial cuidado en la población pediátrica porque $80 \%$ de la exposición solar a lo largo de la vida ocurre antes de los 18 años. ${ }^{24}$

Las medidas de protección solar incluyen a las físicas para reducir la exposición solar ${ }^{25}$ y comprenden: evitar la exposición prolongada a la luz solar, buscar la sombra, usar sombrero de ala ancha y lentes oscuros. ${ }^{26,27}$ Se recomienda cubrir la mayor parte del cuerpo con ropa de colores oscuros, que incrementan de 3 a 5 veces el grado de protección del tejido. ${ }^{28-31}$

Los fotoprotectores, recomendados a partir de los seis meses de edad, tienen como función absorber o reflejar la radiación UV, limitando así el daño a la piel. ${ }^{32} \mathrm{Su}$ adecuada aplicación durante la infancia y la adolescencia puede disminuir, considerablemente, la incidencia de cáncer de piel no melanoma en la vida adulta. ${ }^{33}$ Los fotoprotectores deben ser de amplio espectro (UVA y UVB), resistentes al agua, el sudor y la fricción, con un factor de protección solar de al menos 30+ (Cuadro 2). ${ }^{26,34,35}$ 
Cuadro 2. Uso adecuado de fotoprotectores ${ }^{32,36,37}$

Aplicación diaria, en forma uniforme, en todas las áreas expuestas al sol

La primera capa debe aplicarse 30 minutos antes de salir de casa

Aplicación 20 minutos después para:

- Corregir áreas sin adecuada aplicación

- Evitar de 65 a 80\% de los rayos ultravioleta

Volver a aplicarse cada 2 a 4 horas, sobre todo:

- Si hay mayor actividad física

- Después de nadar

- Después de frotarse con una toalla

- Si hay sudor excesivo

\section{Tips para el adolescente}

El lavado de la cara ayuda a remover el exceso de sebo y evitar la obstrucción folicular, por lo que es una medida indispensable en el adolescente para mejorar el aspecto de la piel y prevenir el acné. ${ }^{38}$ Por ello, se recomienda el lavado gentil con un limpiador facial dos veces al día; el lavado intensivo, o más frecuente, puede dañar la barrera cutánea, resecar la piel y empeorar el acné. ${ }^{39-41}$

El afeitado es un proceso que suele ser irritante debido a la interacción entre la piel y la navaja. ${ }^{42}$ Para minimizar el traumatismo e irritación de la piel se recomienda lavar la cara para hidratar el vello y remover el sebo y las células descamadas en la piel y las aperturas foliculares, y aplicarse gel para afeitar que lubrique la zona de afeitado. ${ }^{43} \mathrm{Al}$ finalizar, debe aplicarse un emoliente. ${ }^{43,44}$

La aplicación de cosméticos y maquillaje en los adolescentes está permitida, pero deben ser libres de aceite, no comedogénicos y fáciles de retirar para evitar brotes de acné, ${ }^{45}$ e hipoalergénicos para evitar dermatitis por contacto.

\section{Importancia de seguir las medidas generales}

El seguimiento de las medidas generales permite mantener saludable a la piel y evitar diversas afecciones: quemaduras por el sol, infecciones, irritación por fricción o uso de químicos e, incluso, de diversas formas de dermatitis.

\section{REFERENCIAS}

1. Holloway $\mathrm{S}$, Jones $\mathrm{V}$. The importance of skin care and assessment. Br J Nurs. 2005; 14 (22): 1172-6. https://doi. org/10.12968/bjon.2005.14.22.20167.

2. Chiou YB, Blume-Peytavi U. Stratum corneum maturation: A review of neonatal skin function. Skin Pharmacol Physiol. 2004; 17 (2): 57-66. doi.10.1159/000076015.

3. Mostafa WZ, Hegazy RA. Vitamin D and the skin: Focus on a complex relationship: A review. J Adv Res. 2013; 6 (6): 793-804. https://doi.org/10.1016/j.jare.2014.01.011.

4. Arenas-Guzmán R. Dermatología. 6ạ ed. México: McGrawHill; 2015:5-6.

5. Giusti $F$, et al. Skin barrier, hydration, and $\mathrm{pH}$ of the skin of infants under 2 years of age. Pediatr Dermatol. 2001; 18 (2): 93-6. https://doi.org/10.1046/j.15251470.2001.018002093.x.

6. Stamatas GN, et al. Infant skin microstructure assessed in vivo differs from adult skin in organization and at the ceIlular level. Pediatr Dermatol. 2010;27(2):125-31. https:// doi.org/10.1111/j.1525-1470.2009.00973.x

7. Turgeon EWT. Adolescent skin : How to keep it healthy. Can Fam Physician. 1986; 32:2427-33. PMID. 21267224.

8. Voegeli D. The effect of washing and drying practices on skin barrier function. J Wound Ostomy Cont Nurs. 2008; 35 (1): 84-90. doi: 10.1097/01.WON.0000308623.68582.d7.

9. Baranda L, et al. Correlation between $\mathrm{pH}$ and irritant effect of cleansers marketed for dry skin. Int J Dermatol. 2002; 41 (8): 494-9. https://doi.org/10.1046/j.13654362.2002.01555.x.

10. Korting $\mathrm{HC}$, et al. Influence of repeated washings with soap and synthetic detergents on $\mathrm{pH}$ and resident flora of the skin of forehead and forearm. Results of a cross-over trial in health probationers. Acta Derm Venereol. 1987; 67 (1): 41-7.

11. Korting $\mathrm{HC}$, Braun-Falco O. The effect of detergents on skin pH and its consequences. Clin Dermatol. 1996; 14 (1): 23-7.

12. Goossens A. New cosmetic contact allergens. Cosmetics. 2015;2 (1): 22-32. https://doi.org/10.3390/cosmetics2010022.

13. Sikora $\mathrm{E}$, et al. Nanoemulsions as a form of perfumery products. Cosmetics. 2018; 5 (4): 63. https://doi.org/10.3390/ cosmetics5040063.

14. Huh $\mathrm{CH}$, et al. Biophysical changes after mechanical injury of the stratum corneum in normal skin. Contact Dermatitis. 2002; 46 (1): 24-8. https://doi.org/10.1034/j.16000536.2002.460105.x.

15. Madison KC. Barrier function of the skin: "La raison d'être" of the epidermis. J Invest Dermatol. 2003; 121 (2): 231-41. https://doi.org/10.1046/j.1523-1747.2003.12359.x. 
Sáez-de-Ocariz M, et al. Medidas generales en Dermatología

16. Lodén M. The clinical benefit of moisturizers. J Eur Acad Dermatol Venereol. 2005; 19 (6): 672-7. https://doi. org/10.1111/j.1468-3083.2005.01326.x.

17. Simpson EL, et al. Emollient enhancement of the skin barrier from birth offers effective atopic dermatitis prevention. J Allergy Clin Immunol. 2014; 134 (4): 818-23. https://doi.org/10.1016/j.jaci.2014.08.005.

18. Mobolaji-Lawal M, Nedorost $\mathrm{S}$. The role of textiles in dermatitis: An update. Curr Allergy Asthma Rep. 2015; 15 (4): 17. https://doi.org/10.1007/s11882-015-0518-0.

19. Le Coz CJ (ed), et al. Contact Dermatitis. Berlin: Springer, 2006.

20. Barker RL, et al. In vivo cutaneous and perceived comfort response to fabric: Part II: Mechanical and surface related comfort property determinations for three experimental knit fabrics. Text Res J. 1990; 60 (8): 490-4.

21. Kaynak HK, Çelik Hi. Thermophysiological comfort and performance properties of knitted fabrics produced from different spinning technologies. J Text Inst. 2018; 109 (4): 536-42.

22. Markee NL, et al. In vivo cutaneous and perceived comfort response to fabric: Part IV: Perceived sensations to three experimental garments worn by subjects exercising in a hot, humid environment. Text Res J. 1990; 60 (10): 561-8.

23. Vitols $P$, Oates RK. Teaching children about skin cancer prevention: why wait for adolescence? Aust N Z J Public Health. 1997; 21 (6): 602-5. https://doi.org/10.1111/ j.1467-842X.1997.tb01763.x.

24. Pustišek N, et al. Acute skin sun damage in children and its consequences in adults. Coll Antropol. 2010; 34 (Suppl 2): 233-7. https://hrcak.srce.hr/51665.

25. Gilaberte $Y$, et al. Fotoprotección. Actas Dermosifiliogr. 2003; 94 (5): 271-93. https://doi.org/10.1016/S00017310(03)76688-5.

26. Quatrano NA, Dinulos JG. Current principles of sunscreen use in children. Curr Opin Pediatr. 2013; 25 (1): 122-9. doi: 10.1097/MOP.0b013e32835c2b57.

27. World Health Organization, World Meteorological Organization, United Enviroment Programme IC on N-IRP. Global solar UV index : a practical guide, 2002. http:// www.who.int/uv/.

28. Downs NJ, Harrison SL. A comprehensive approach to evaluating and classifying sun-protective clothing. $\mathrm{Br} J \mathrm{Dermatol}$. 2018; 178 (4): 958-64. https://doi.org/10.1111/bjd.15938.

29. Rossi JS, et al. Preventing skin cancer through behavior change. Implications for interventions. Dermatol Clin. 1995;13 (3): 613-22.

30. Hoffmann K, et al. Defined UV protection by apparel textiles. Arch Dermatol. 2001; 137 (8): 1089-94. doi:10-1001/ pubs.Arch Dermatol.-ISSN-0003-987x-137-8-dre10006.
31. Wesson KM, Silverberg NB. Sun protection education in the United States: What we know and what needs to be taught. Cutis. 2003; 71 (1): 71-4.

32. Rai R, et al. Update on photoprotection. Indian J Dermatol. 2012; 57 (5): 335-42. 10.4103/0019-5154.100472.

33. Heckman CJ, Coups EJ. Correlates of sunscreen use among high school students: a cross-sectional survey. BMC Public Health. 2011; 11 (1): 679. doi.10.1186/1471-2458-11-679. https://doi.org/10.1186/1471-2458-11-679.

34. Valdivielso-Ramos M, Herranz JM. Actualización en fotoprotección infantil. An Pediatr. 2010; 72 (4): 282.e1-282.e9. https://doi.org/10.1016/j.anpedi.2009.06.013.

35. Williams JD, et al. SPF $100+$ sunscreen is more protective against sunburn than SPF 50+ in actual use: Results of a randomized, double-blind, split-face, natural sunlight exposure clinical trial. J Am Acad Dermatol. 2018; 78 (5): 902-10. https://doi.org/10.1016/j.jaad.2017.12.062.

36. Lowe NJ. Photoprotection. Semin Dermatol. 1990; 9 (1): 78-83. PMID: 2203447

37. Diffey BL, Grice J. The influence of sunscreen type on photoprotection. Br J Dermatol. 1997; 137 (1): 103-5. https://doi.org/10.1046/j.1365-2133.1997.17761863.x.

38. Isoda K, et al. Effects of washing of the face with a mild facial cleanser formulated with sodium laureth carboxylate and alkyl carboxylates on acne in Japanese adult males. Ski Res Technol. 2015; 21 (2): 247-53. https://doi.org/10.1111/ srt.12183.

39. Sakuma TH, Maibach HI. Oily skin: An overview. Skin Pharmacol Physiol. 2012; 25 (5): 227-35. https://doi. org/10.1159/000338978.

40. Mukhopadhyay P. Cleansers and their role in various dermatological disorders. Indian J Dermatol. 2011; 56 (1): 2-6. https://www.ncbi.nlm.nih.gov/sars-cov-2/.

41. Choi JM, et al. A single-blinded, randomized, controlled clinical trial evaluating the effect of face washing on acne vulgaris. Pediatr Dermatol. 2006; 23 (5): 421-7. https://doi. org/10.1111/j.1525-1470.2006.00276.x.

42. Kubba, et al. Cosmetics and skin care in acne. Indian J Dermatology Venereol Leprol. 2009; 75 (7): 55.

43. Draelos ZD. Male skin and ingredients relevant to male skin care. Br J Dermatol. 2012; 166 (Suppl 1): 13-6. https://doi. org/10.1111/j.1365-2133.2011.10784.x.

44. Oblong JE. Male skin care: Shaving and moisturization needs. Dermatol Ther. 2012; 25 (3): 238-43. https://doi. org/10.1111/j.1529-8019.2012.01502.x.

45. Goodman G. Cleansing and moisturizing in acne patients. Am J Clin Dermatol. 2009; 10 (1): 1-6. https://doi. org/10.2165/0128071-200910001-00001. 